\title{
Corrélations entre les caractéristiques phénotypiques et détermination d'une formule barymétrique chez le taurin Kouri du Niger
}

\author{
Moustapha Gréma 1,3, Hamza Seydou Korombé2, Albert Soudré4, Stéphane A.R. Tapsoba5, Maaouia \\ Mahaman Abdou Moussa ${ }^{6}$, Moumouni Sanou ${ }^{5}$, Amadou Traorés ${ }^{*}$, Moumouni Issa ${ }^{1}$, Hamani Marichatou 6 , \\ Moussa Assane ${ }^{7}$, Hamidou H. Tamboura ${ }^{5}$ et Yenikoye Alhassane ${ }^{6}$ \\ 1 Université Abdou Moumouni de Niamey, Faculté des Sciences et Techniques, BP 10662 Niamey, Niger \\ ${ }^{2}$ Centre de Multiplication du Bétail du Niger, BP : 827 Niamey, Niger \\ 3Université de Diffa, Faculté des Sciences Agronomiques, BP 78, Diffa, Niger \\ 4Université Norbert Zongo (UNZ) de Koudougou, Unité de Formation et de Recherches en Sciences et \\ Technologies, BP 376 Koudougou, Burkina Faso \\ 5Institut de l'Environnement et de Recherches Agricoles (INERA), Laboratoire de Biologie et santés animales, $04 \mathrm{BP}$ \\ 8645, Ouagadougou 04, Burkina Faso \\ 6Université Abdou Moumouni de Niamey, Faculté d'Agronomie, BP 10960 Niamey, Niger \\ ${ }^{7}$ EISMV Laboratoire de physiologie et de pharmacodynamie, BP n 5077 Dakar - Fann, SENEGAL \\ * Auteur correspondant: Dr Amadou TRAORE, DVM, PhD, Institut de l'Environnement et de Recherches Agricoles \\ (INERA), Laboratoire de Biologie et santés animales, 04 BP 8645, Ouagadougou 04, Burkina Faso. Email : \\ traore_pa@yahoo.fr
}

Original submitted in on $8^{\text {th }}$ August 2018. Published online at www.m.elewa.org on $30^{\text {th }}$ November 2018 https://dx.doi.org/10.4314/jab.v131i1.7

\section{RÉSUMÉ}

Objectif : Cette étude vise à contribuer à la connaissance des caractéristiques phénotypiques du taurin Kouri du Niger.

Méthodologie et résultats : Les caractéristiques phénotypiques du taurin Kouri du Niger ont fait l'objet d'une étude conduite dans la région de Diffa au niveau des sites situés aux alentours de Kinjandi dans la commune de Gueskerou et au Centre Secondaire de Multiplication du Bétail de Sayam. Des mensurations ont été effectuées directement sur des animaux et ont permis d'enregistrer des paramètres quantitatifs. Des paramètres qualitatifs ont été renseignés sur la base de l'observation des animaux. Un total de 475 animaux (423 femelles, 36 mâles entiers et 16 mâles castrés) âgés d'au moins 4 ans a été concerné par l'étude. Les moyennes des paramètres phénotypiques quantitatifs suivants ont été notées : Hauteur au garrot $(\mathrm{HG})$ : $125,65 \pm 6,08 \mathrm{~cm}$; Périmètre thoracique (PT) : 157,95 $\pm 10 \mathrm{~cm}$; Hauteur au sacrum (HS) : 133,50 $\pm 6,10 \mathrm{~cm}$; Largeur aux ischions (LI) : 14,41 $\pm 2,75$. Des différences significatives ont été observées entre les sexes. Le PT est le paramètre le plus corrélé au poids avec un coefficient de corrélation de 0,843 . Trois modèles barymétriques sont proposés et ont permis de noter des coefficients d'ajustement de $R_{1}{ }^{2}=0,79 ; R_{2}{ }^{2}=0,74$ et $\mathrm{R}_{3}{ }^{2}=0,71$. Les paramètres phénotypiques qualitatifs ont fait ressortir un profil rectiligne dominant $(85,86 \%)$; des oreilles à orientation latérale $(99,79 \%)$; des cornes présentes $(97,89 \%)$ et fixées $(97,41 \%)$, un dos rectiligne chez $86,50 \%$ des animaux. Aussi la couleur blanche de la robe domine avec 50,84\%. 
Conclusion et application des résultats : En somme, le taurin Kouri du Niger peut être considéré comme une race assez hétérogène sur le plan phénotypique. Cette variabilité pourrait être favorable à une sélection en vue d'une amélioration génétique de la race.

Mots clés : phénotype, formule barymétrique, taurin Kouri, Niger.

\section{ABSTRACT \\ Correlations between Phenotypic measurements and determination of a Barymetric Formula in Niger Kuri cattle breed.}

Objective: The main objective of this study is to contribute to increase knowledge on Niger Kuri phenotypic characteristics.

Methodology and Results: Phenotypic characteristics of Niger Kuri cattle breed have been assessed in the region of Diffa, Niger, including sampling sites around Kinjandi in Gueskerou commune, and in the Sayam Cattle Secondary Multiplication Center. Measurements were made directly on animals and allowed to record quantitative parameters. Qualitative parameters were given based on the observation of the animals. A total of 475 individuals ( 423 females, 36 males and 16 castrated males) with at least 4 years age have been sampled. The means values of phenotypic traits measured were as follow: quantitative phenotypic parameters were measured. Height at withers: $125.65 \pm 6.08 \mathrm{~cm}$, Heart girth: $157.95 \pm 10 \mathrm{~cm}$, height at hips: $133.50 \pm 6.10 \mathrm{~cm}$, Ischium width: $14.41 \pm 2.75$. Significant differences were observed between sexes. Heart girth is the most correlated parameter to the weight with a correlation coefficient of 0.843 . Three barymetric models were used and showed adjusted coefficients of $\mathrm{R}_{1}{ }^{2}=0.79, \mathrm{R}_{2}{ }^{2}=0.74$ and $\mathrm{R}_{3}{ }^{2}=0.71$. The qualitative phenotypic parameters revealed à dominant straight profile $(85.86 \%)$, lateral oriented ears $(99.79 \%)$, presence of horns $(97.89 \%)$ and fixed $(97.41 \%)$, straight back $(86.50 \%)$ among the individuals. Besides, the white color pattern is predominant $(50.84 \%)$.

Conclusion and application of results: Overall, Niger Kuri cattle breed may be considered as heterogeneous breed at phenotypic level. This variability may be favorable for selection strategy for this valuable breed improvement.

Keywords: phenotype, barymetric formula, Kuri, Niger.

\section{INTRODUCTION}

Pays au trois quart désertique, et de plus en plus régulièrement confronté à la sécheresse, le Niger vit pourtant presqu'exclusivement de l'agriculture et de l'élevage (APESS, 2014). Activité de grande importance, l'élevage emploie près de $87 \%$ de la population active du pays et représente, pour l'économie, la composante la plus dynamique et porteuse de croissance du secteur primaire. En effet, en 2010, les productions animales ont contribué pour près de $11 \%$ au Produit Intérieur Brut (PIB) et 24\% au PIB agricole, et se placent au premier rang des recettes totales d'exportation des produits agrosylvo-pastoraux avec $22 \%$. L'élevage contribue également à plus de $15 \%$ au budget des ménages, et assure la satisfaction des besoins alimentaires, à hauteur de $25 \%$. II constitue un pourvoyeur de recettes pour l'État et les collectivités territoriales
(Niger, 2013). L'effectif total du cheptel est estimé en 2013 à 39.413 .000 têtes dont 10.733 .000 têtes de bovins (INS, 2014). Le cheptel bovin se compose de cinq races dont quatre races de zébu (Azawak, Bororo, Djelli, Goudali) et une race taurine, le Kouri (Rhissa, 2010). Ce dernier est une race stabilisée. Son berceau se situe au lac Tchad (Queval et al., 1971). Le taurin Kouri est intéressant en raison de ses particularités biologiques de résistance innée aux pathologies tropicales redoutables (Tawah et al., 1997). Ses cornes flottantes, mises à profit lors des traversées du Lac-Tchad, constituent une caractéristique de cet animal. Sa docilité et sa bonne performance laitière sont prisées par les éleveurs (Zeuh et al., 2014). Mais aujourd'hui, la race Kouri est menacée d'extinction à cause de la dégradation de son biotope naturel constitué des îles et berges 
du lac Tchad, mais aussi le croisement avec des bovins zébus (Bourzat et al., 1992 ; Tchad., 2003 ; Mian., 2008; Marichatou et al., 2011). Par conséquent des actions de protection et de préservation en sa faveur doivent être intensifiées. C'est ainsi que pour la conservation in situ de cette race bovine, les pouvoirs publics du Niger ont mis en place depuis 1977 le Centre Secondaire de Multiplication de Bétail (CSMB) de Sayam dont l'activité essentielle est la sélection et la multiplication du taurin Kouri. Cependant, contrairement au zébu Azawak qui a fait l'objet de plusieurs études tant sur les plans morphologiques que zootechniques, le taurin Kouri du Niger n'est pas bien connu sur le plan scientifique. Au Niger, la race Kouri n'a pas fait l'objet d'investigations approfondies sur le plan scientifique (Marichatou et al., 2011), comparativement à la même race au Tchad (Queval

\section{MATÉRIEL ET MÉTHODES}

Zone d'étude : La région de Diffa où l'étude a été menée, est située à l'extrême Est du Niger et est comprise entre $10^{\circ} 30^{\prime}$ et $5^{\circ} 35^{\prime}$ de longitude Est, $13^{\circ} 04^{\prime}$ et $18^{\circ} 00^{\prime}$ de latitude Nord. Arrosée par le lac Tchad et la Komadougou, cette région est limitée à l'Est par le Tchad, au Sud par le Nigéria, au Nord par la région d'Agadez et à l'Ouest par la région de Zinder (INS, 2015; FAO, 2003a). Le relief est très peu accentué, le point le plus bas étant le lac Tchad à $275 \mathrm{~m}$ d'altitude et les points les plus hauts sont représentés par les massifs crétacés de Termit et Agadem (altitude supérieure à $500 \mathrm{~m}$ ), dans le Nord. A l'Ouest, le bassin est limité par les affleurements cristallins du Mounio et le massif crétacé du Koutous. L'essentiel du paysage est constitué de grandes et al., 1971, Queval et Petit, 1973; Souvenir et Zeuh., 1996 ; Souvenir et al., 1999 ; Tawah et al., 1997 ; Zeuh et al., 2014 ; Tellah et al., 2015). Face à cette situation, une question importante est de savoir si le taurin Kouri du Niger est une race homogène sur le plan phénotypique ? En effet selon la FAO (2013), la caractérisation phénotypique est essentielle pour la planification de la gestion des ressources zoo génétiques aux niveaux local, national, régional et mondial. Les paramètres phénotypiques quantitatifs étant directement corrélés aux paramètres de production, leur connaissance est importante pour la maîtrise et l'amélioration des performances de production des animaux. C'est dans ce contexte que cette étude a été menée avec comme objectif spécifique de caractériser sur le plan phénotypique le taurin Kouri du Niger.

étendues monotones de sédiments alluviaux et éoliens quaternaires (Christian et al., 1998). Le climat est de type sahélien dans la partie Sud, et sahélo-saharien au Nord. II se caractérise par une longue saison sèche de Novembre à Mai et une saison humide de Juin à Octobre. La pluviométrie y décroît rapidement du Sud au Nord, et les précipitations annuelles moyennes sont inférieures à $400 \mathrm{~mm}$ sur l'ensemble de la région. La région a une vocation pastorale (FAO, 2003b).

Collecte des données: La collecte des données a concerné les troupeaux autour de Kinjandi et au niveau du centre secondaire de multiplication du bétail de Sayam (Figure 1). 


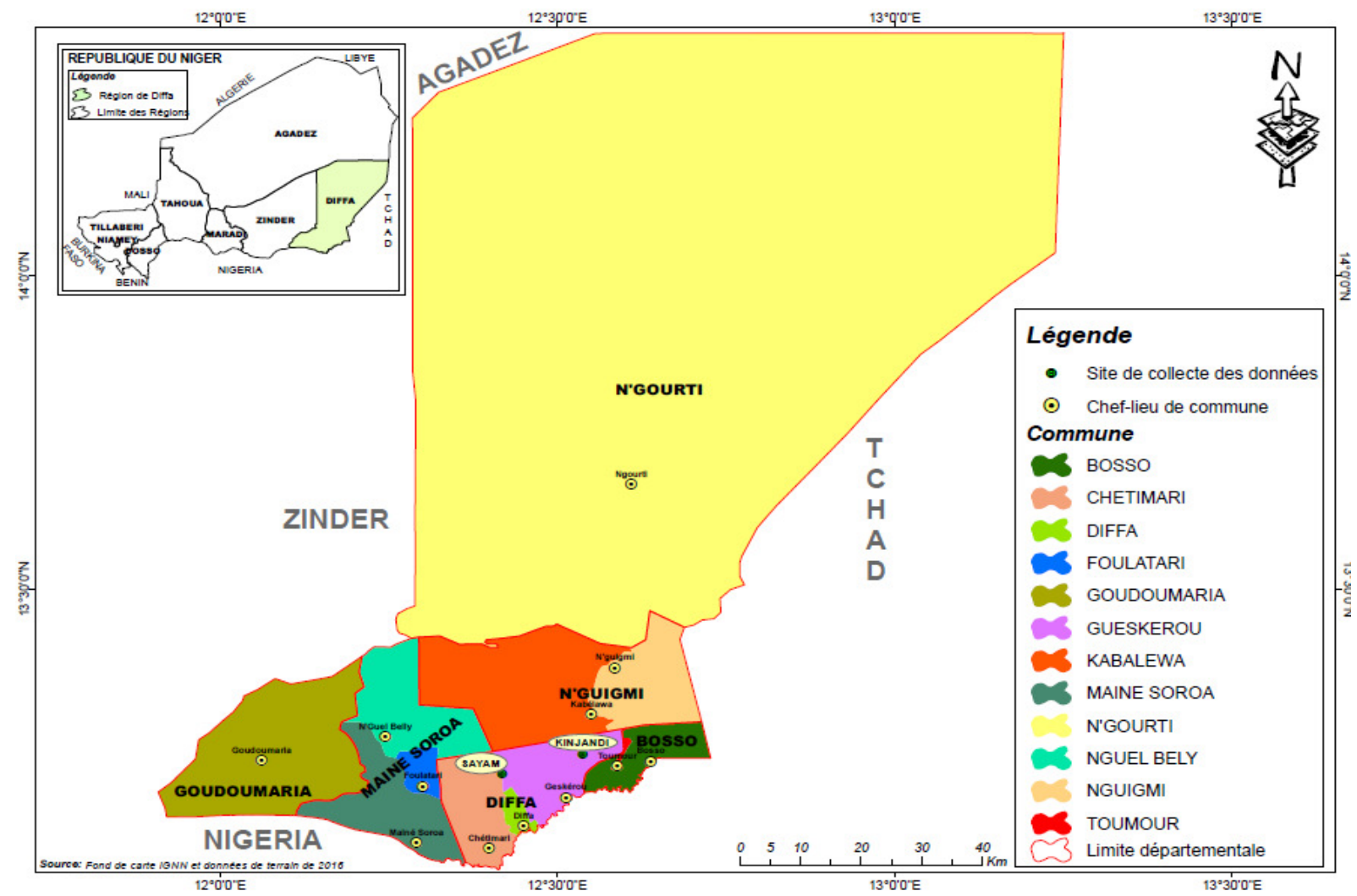

Figure 1 : Localisation des sites de collecte des données

Au total 475 animaux de race Kouri, âgés d'au moins 4 ans, ont été utilisés pour cette étude. Cet échantillon se répartit en 423 femelles, 36 mâles entiers et 16 mâles castrés. II faut noter également que dans chaque troupeau, au maximum 5 animaux ont été utilisés tout en tenant compte de la taille du troupeau et des liens de parenté entre les animaux. Les informations recherchées sont d'ordre qualitatif et quantitatif. Les caractères qualitatifs concernent l'état physique, la forme, la couleur de la robe et l'apparence externe des animaux (profil de la tête, orientation de l'oreille, forme de l'oreille, orientation des cornes, appréciation de la ligne du dos, etc.). Ainsi pour chaque animal, ces caractères ont été renseignés sur la base de l'observation. Aussi pour chaque animal échantillonné 4 photos ( 1 de face, 2 de profil, et 1 de derrière) ont été prises en vue de son identification complète et précise en cas de doute. Les mesures de hauteur ont été effectuées et ont concerné la hauteur au garrot, la hauteur au sacrum, la profondeur de la poitrine ou hauteur de la poitrine. Les mesures de longueur ont porté sur la longueur scapulo-ischiale, la longueur du bassin, la longueur de la tête. Les mesures de largeur réalisées sont la largeur aux ischions ou largeur aux fesses, la largeur aux hanches, la largeur aux épaules, la largeur de la tête.

Établissement d'une formule barymétrique : Plusieurs formules d'ajustement concernant l'estimation du poids vif sont proposées dans la littérature. Elles prennent en considération diverses mensurations: périmètre thoracique, hauteur au garrot, longueur scapulo-ischiale, etc. et divers facteurs de variation comme l'âge, le sexe et le niveau d'alimentation (Delage et al., 1955 ; Pagot et Delaine, 1959 ; Chollou et al., 1978 ; Poivey et al., 1980, Dineur et Thys, 1986 ; Dodo et al., 2001). Pour déterminer une formule barymétrique applicable au taurin Kouri du Niger, le choix s'est porté sur les paramètres corporels qui ont une bonne corrélation avec le poids (Buldgen et al., 1984). II s'agit du périmètre thoracique (PT), de la hauteur au garrot (HG), de la hauteur au sacrum (HS), de la longueur scapulo-ischiale (Lsi), de la longueur du corps (LC) et de la profondeur poitrine (Pp). Analyses statistiques des données: Les données recueillies ont été saisies sur Excel. Le logiciel SPSS 20.0 a permis de calculer les moyennes des variables quantitatives et de réaliser des tests ANOVA et post hoc pour comparer les moyennes entre les groupes. Pour les 
variables qualitatives, des tableaux croisés en fonction du sexe ont été réalisés avec SPSS 20.0. Le logiciel XLSTAT 2014 a été utilisé pour faire des tests de corrélation avec l'analyse en composante principale (ACP) et pour affiner les résultats, une analyse factorielle discriminante a été effectuée (AFD). XLSTAT 2014 a aussi servi pour faire des régressions linéaires en vue de déterminer des formules barymétriques. Ainsi, trois séries de régression linéaire ont été réalisées en vue de retenir la meilleure régression pour l'estimation du poids.

La première a pris en compte tous les six paramètres cités ci-dessus, en fonction de la droite de régression de

\section{RÉSULTATS}

\section{Paramètres morpho biométriques et leurs} corrélations

Valeurs moyennes des paramètres corporels quantitatifs : Le tableau 1 présente les moyennes des paramètres corporels quantitatifs ainsi que les résultats de leur comparaison selon le sexe. II ressort de l'analyse de ce tableau, des différences significatives entre les moyennes de tous les groupes (Mâle (M), Femelle (F), Castré (C)) pour la hauteur au garrot $\left(\mathrm{HG}_{\mathrm{C}}>\mathrm{HG}_{\mathrm{M}}>\mathrm{HG}_{\mathrm{F}}\right)$; le périmètre thoracique $\left(P T_{C}>P T_{M}>P T_{F}\right)$; la hauteur au sacrum $\left(\mathrm{HS}_{\mathrm{C}}>\mathrm{HS}_{\mathrm{M}}>\mathrm{HS}_{\mathrm{F}}\right)$ et la largeur aux ischions $\left(\mathrm{l}_{\mathrm{F}}>\mathrm{li}_{\mathrm{C}}>\mathrm{l}_{\mathrm{M}}\right)$. Des différences significatives ont également type : $P=a_{1}+b_{1}{ }^{*} \mathrm{LC}+\mathrm{c}_{1}{ }^{*} \mathrm{HG}+\mathrm{d}_{1}{ }^{*} \mathrm{PT}+\mathrm{e}_{1}{ }^{*} \mathrm{HS}+\mathrm{f}_{1}{ }^{*} \mathrm{Ls}+\mathrm{g}_{1}{ }^{*} \mathrm{Pp} . \mathrm{a}_{1}$, $b_{1}, c_{1}, d_{1}, e_{1}, f_{1}$ et $g_{1}$, sont des constantes.

Dans la deuxième série, seuls trois paramètres ont été utilisés, il s'agit du PT, de la HG et de la HS, en fonction de la droite de régression de type : $\mathrm{P}=$ $a_{2}+b_{2}{ }^{*} H G+c_{2}{ }^{*} P T+d_{2}{ }^{*} H S . \quad a_{2}, \quad b_{2}, c_{2}$ et $d_{2}$ sont des constantes

La troisième régression linéaire n'a pris en compte que le $\mathrm{PT}$, en fonction de la droite de régression de type : $\mathrm{P}=$ $a_{3}+b_{3}{ }^{*} P T . a_{3}$ et $b_{3}$ sont des constantes.

II est à noter que le niveau de significativité est de 5\% pour tous les tests réalisés.

été observées entre les moyennes des mâles toutes catégories confondues (mâles entiers et mâle castrés) et celles des femelles pour les paramètres suivants: la longueur scapulo-ischiale $\left(\mathrm{Lsi}_{M}=\mathrm{Lsi}_{\mathrm{C}}, \mathrm{Ls}_{\mathrm{iC}}>\mathrm{Lsi}_{\mathrm{F}}\right)$, ainsi que pour le tour du Jarret (TJ), la largeur aux hanches (lh), la largeur aux épaules (le), la longueur de la queue $(L Q)$, la profondeur de la poitrine (Pp) et enfin le poids (P). Aussi l'analyse du tableau permet de montrer que pour la hauteur au garrot $(H G)$, le périmètre thoracique (PT), la hauteur au sacrum (HS) et la largeur aux ischions (ii) le dimorphisme sexuel est très marqué. 
Gréma et al., J. Appl. Biosci. 2018 Corrélations entre les caractéristiques phénotypiques et détermination d'une formule barymétrique chez le taurin Kouri du Niger

Tableau 1: Moyennes et Écart-types des paramètres corporels quantitatifs

\begin{tabular}{|c|c|c|c|c|c|c|c|c|c|c|c|c|c|c|c|c|}
\hline Sexe & & $\mathbf{N}$ & HG & PT & HS & LC & Lsi & LB & li & $\mathrm{TJ}$ & Lh & le & LQ & Ip & $\mathrm{Pp}$ & $\mathbf{P}$ \\
\hline \multirow{2}{*}{ Mâle } & Moyenne & 36 & $129,47^{a}$ & $163,17^{a}$ & $137,18^{a}$ & $101,42^{a b c}$ & $155,06^{\mathrm{ac}}$ & $40,92^{\mathrm{abc}}$ & $11,21^{a}$ & $37,07^{\mathrm{ac}}$ & $46,44^{a b}$ & $52,78^{a b c}$ & $143,50^{\text {ac }}$ & $24,36^{a b c}$ & $74,22^{\mathrm{ac}}$ & $348,25^{\mathrm{ac}}$ \\
\hline & Écart-type & & 5,02 & 11,39 & 4,61 & 10,88 & 13,76 & 3,07 & 2,08 & 3,67 & 4,23 & 4,45 & 13,10 & 3,37 & 5,84 & 77,28 \\
\hline \multirow{2}{*}{ Femelle } & Moyenne & 423 & $124,96^{b}$ & $157,03^{b}$ & $132,82^{b}$ & $98,51^{\mathrm{bac}}$ & $150,13^{b}$ & $42,07^{\mathrm{bac}}$ & $14,71^{b}$ & $34,81^{b}$ & $46,31^{\text {ba }}$ & $51,26^{\text {ba }}$ & $133,68^{b}$ & $24,25^{\text {bac }}$ & $71,10^{b}$ & $310,40^{\mathrm{b}}$ \\
\hline & Écart-type & & 5,79 & 9,63 & 5,84 & 9,10 & 11,10 & 3,37 & 2,65 & 2,48 & 4,04 & 4,73 & 12,05 & 4,09 & 5,10 & 51,84 \\
\hline \multirow{2}{*}{ Castré } & Moyenne & 16 & $134,59^{c}$ & $169,13^{c}$ & $143,19 c$ & $101,25^{c a b}$ & 159,06 ca & 42,63 cab & $13,25^{c}$ & $38,19 \mathrm{ca}$ & $49,25^{c}$ & 55,38 са & $146,16^{\mathrm{ca}}$ & $25,19 \mathrm{cab}$ & $76,75^{\text {ca }}$ & $370^{\text {ca }}$ \\
\hline & Écart-type & & 6,44 & 8,65 & 5,10 & 4,91 & 10,63 & 4,06 & 1,69 & 2,45 & 4,75 & 3,96 & 12,13 & 1,80 & 3,59 & 51,12 \\
\hline \multirow{2}{*}{ Race Kouri } & Moyenne & 475 & 125,65 & 157,945 & 133,50 & 98,86 & 150,82 & 42,01 & 14,41 & 35,10 & 46,44 & 51,53 & 135,11 & 24,29 & 71,56 & 314,38 \\
\hline & Écart-type & & 6,08 & 10,00 & 6,10 & 9,15 & 11,45 & 3,36 & 2,75 & 2,71 & 4,07 & 4,74 & 11,23 & 3,98 & 5,24 & 54,36 \\
\hline
\end{tabular}

$\mathrm{N}$ : taille de l'échantillon ; HG : hauteur au garrot ; PT : périmètre thoracique ; HS : hauteur au sacrum ; LC : longueur du corps ; Lsi : longueur scapulo-ischiale ; LB : longueur du bassin ; li : largeur aux ischions ; TJ : le tour du Jarret; Ih : largeur aux hanches; $;$ : : largeur aux épaules ; $L Q:$ longueur de la queue $; \mathrm{Pp}:$ profondeur de la poitrine $; \mathrm{P}: \mathrm{poids}$.

NB : Dans la même colonne, deux moyennes portant des lettres différentes $(a, b$, ou $c)$ sont statistiquement différentes $(P<0,05)$.

\begin{tabular}{|c|c|c|c|c|c|c|c|c|c|c|c|c|c|c|c|c|c|c|}
\hline Variables & $\mathrm{LC}$ & HG & PT & HS & Lsi & $\mathrm{Pp}$ & Ip & LQ & le & Ih & li & LB & $\mathrm{TJ}$ & $\mathbf{P}$ & Âges & $\mathrm{C}$ & $F$ & $M$ \\
\hline LC & 1,00 & & & & & & & & & & & & & & & & & \\
\hline HG & 0,66 & 1,00 & & & & & & & & & & & & & & & & \\
\hline PT & 0,55 & 0,74 & 1,00 & & & & & & & & & & & & & & & \\
\hline HS & 0,62 & 0,91 & 0,73 & 1,00 & & & & & & & & & & & & & & \\
\hline Lsi & 0,61 & 0,58 & 0,51 & 0,54 & 1,00 & & & & & & & & & & & & & \\
\hline $\mathrm{Pp}$ & 0,41 & 0,51 & 0,65 & 0,53 & 0,41 & 1,00 & & & & & & & & & & & & \\
\hline Ip & 0,31 & 0,31 & 0,43 & 0,31 & 0,23 & 0,24 & 1,00 & & & & & & & & & & & \\
\hline LQ & 0,48 & 0,57 & 0,45 & 0,55 & 0,43 & 0,35 & 0,19 & 1,00 & & & & & & & & & & \\
\hline le & 0,31 & 0,39 & 0,33 & 0,28 & 0,33 & 0,40 & 0,23 & 0,25 & 1,00 & & & & & & & & & \\
\hline Ih & 0,39 & 0,50 & 0,61 & 0,55 & 0,42 & 0,51 & 0,19 & 0,33 & 0,23 & 1,00 & & & & & & & & \\
\hline li & 0,34 & 0,33 & 0,31 & 0,34 & 0,21 & 0,16 & 0,25 & 0,22 & 0,10 & 0,23 & 1,00 & & & & & & & \\
\hline LB & 0,40 & 0,40 & 0,46 & 0,45 & 0,44 & 0,41 & 0,11 & 0,34 & 0,27 & 0,42 & 0,33 & 1,00 & & & & & & \\
\hline TJ & 0,46 & 0,65 & 0,55 & 0,67 & 0,43 & 0,43 & 0,34 & 0,44 & 0,25 & 0,43 & 0,29 & 0,36 & 1,00 & & & & & \\
\hline P & 0,68 & 0,74 & 0,84 & 0,71 & 0,60 & 0,60 & 0,43 & 0,55 & 0,38 & 0,60 & 0,38 & 0,46 & 0,60 & 1,00 & & & & \\
\hline Âges & 0,32 & 0,32 & 0,42 & 0,27 & 0,39 & 0,27 & 0,07 & 0,30 & 0,21 & 0,40 & 0,21 & 0,39 & 0,15 & 0,44 & 1,00 & & & \\
\hline C & 0,11 & 0,33 & 0,27 & 0,32 & 0,13 & 0,28 & 0,04 & 0,25 & 0,22 & 0,13 & $-0,12$ & 0,02 & 0,21 & 0,22 & $-0,07$ & 1,00 & & \\
\hline $\mathrm{F}$ & $-0,19$ & $-0,36$ & $-0,30$ & $-0,33$ & $-0,13$ & $-0,28$ & $-0,05$ & $-0,26$ & $-0,22$ & $-0,10$ & 0,22 & 0,06 & $-0,24$ & $-0,27$ & 0,13 & $-0,75$ & 1,00 & \\
\hline M & 0,16 & 0,16 & 0,14 & 0,12 & 0,04 & 0,09 & 0,03 & 0,10 & 0,08 & 0,01 & $-0,20$ & $-0,12$ & 0,11 & 0,15 & $-0,12$ & $-0,04$ & $-0,63$ & 1,00 \\
\hline
\end{tabular}

LC : longueur du corps ; HG : hauteur au garrot ; PT : périmètre thoracique ; HS : hauteur au sacrum ; Lsi : longueur scapulo-ischiale ; Pp : profondeur de la poitrine ; LQ : longueur de la queue ; le : largeur aux épaules ; Ih :largeur aux hanches ; li : largeur aux ischions ; LB : longueur du bassin ; TJ : le tour du Jarret ; P : poids ; C : Castré ; F : Femelle ; M : Mâle. 
Corrélations entre les paramètres corporels quantitatifs: Dans le tableau 2, sont consignés les coefficients de corrélation entre les différents paramètres corporels quantitatifs en variables principales mais aussi ceux de l'âge et du sexe en variables supplémentaires. L'analyse de ces résultats permet de mettre en évidence l'existence de corrélations entre certaines variables. Selon les résultats du tableau, trois types de corrélations se distinguent: les fortes corrélations positives enregistrées entre $\mathrm{HG}$ et $\mathrm{HS}(0,91)$ et entre $\mathrm{PT}$ et $\mathrm{P}(0,84)$
; les corrélations moyennes positives (coefficients de corrélation compris entre 0,5 et 0,7 ) apparaissent entre $P$, HG, LC, Lsi, Pp, LQ, Ih, TJ, PT et HS et les corrélations faibles positives et négatives (coefficient de corrélation inférieur à 0,5) apparaissent en majorité entre les variables principales et supplémentaires. La figure 2 montre la répartition des variables principales en rouge et des variables supplémentaires en bleu dans le plan factoriel $\left(\mathrm{F}_{1} X \mathrm{~F}_{2}\right)$.

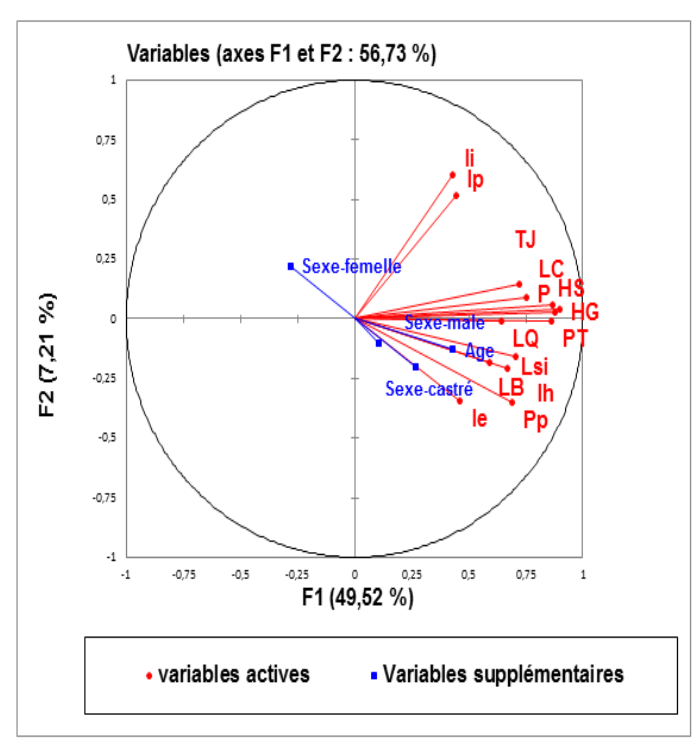

Figure 2: Cercle de corrélation des paramètres corporels quantitatifs donné par l'ACP

LC: longueur du corps; HG : hauteur au garrot; PT : périmètre thoracique ; HS : hauteur au sacrum ; Lsi : Iongueur scapulo-ischiale ; $\mathrm{Pp}$ : profondeur de la poitrine $L Q$ : longueur de la queue ; le : largeur aux épaules ; Ih : largeur aux hanches ; li : largeur aux ischions ; LB : longueur du bassin ; TJ : le tour du Jarret ; $P$ : poids.

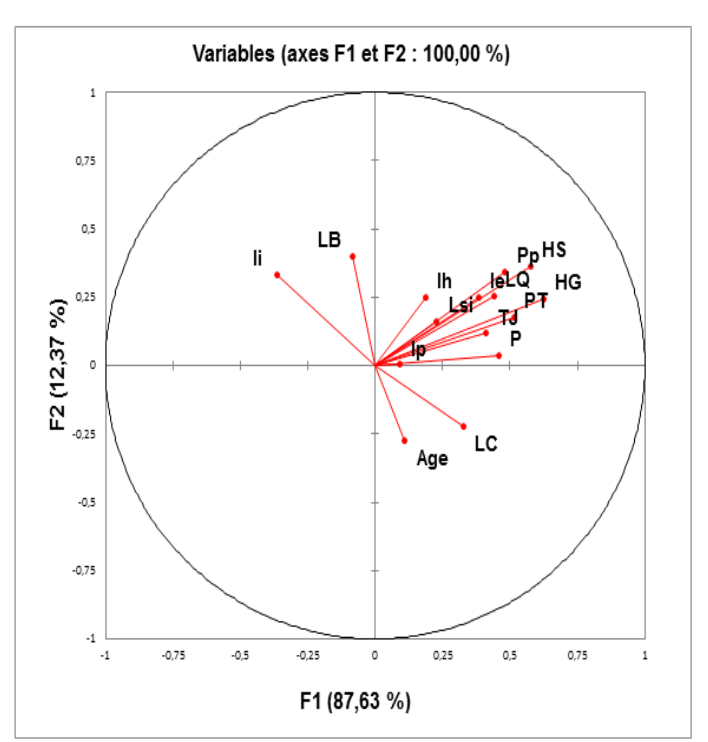

Figure 3: Cercle de corrélation des paramètres corporels quantitatifs donné par l'AFD

LC : longueur du corps ; HG : hauteur au garrot ; PT : périmètre thoracique $\mathrm{HS}$ : hauteur au sacrum ; Lsi : longueur scapulo-ischiale; $\mathrm{Pp}$ : profondeur de la poitrine ; LQ : longueur de la queue ; le : largeur aux épaules ; Ih : largeur aux hanches ; li : largeur aux ischions ; LB : longueur du bassin ; TJ : le tour du Jarret ; $P$ : poids.

race, comme en témoignent les fortes corrélations positives entre eux, les mâles et les castrés et les fortes corrélations négatives entre eux et les femelles. Les figures 3 et 4 présentent les résultats de l'AFD. L'AFD donne un rapport de $94,06 \%$ pour le nombre d'observations bien classées (données non présentées). La figure 4 donne la répartition des individus dans le plan factoriel $\left(F_{1} X F_{2}\right)$ en fonction des paramètres quantitatifs corporels. On observe selon les barycentres de chaque groupe que les femelles sont bien discriminées sur les deux axes $\left(F_{1}, F_{2}\right)$, tandis que les mâles et les castrés sont discriminés sur l'axe $F_{2}$. II ressort de l'analyse de la figure que trois groupes distincts d'individus (Mâle, 
Femelle, Castré) se forment. Cependant, ces groupes ne sont pas totalement distincts, il existe un sous-groupe intermédiaire. Conformément à l'ACP (Figure 2), la figure 3 de l'AFD permet de déduire que les individus échantillonnés se distinguent en fonction du sexe. Ainsi les individus mâles sont mieux discriminés par l'importance de la longueur du corps, la hauteur au garrot, le tour du jarret et le poids alors que, pour les individus castrés ce sont : le périmètre thoracique, la hauteur au sacrum, la profondeur de la poitrine, la longueur de la queue, la largeur aux épaules et la longueur scapuloischiale, et enfin pour les femelles, la largeur de la poitrine, la largeur aux hanches, la largeur aux ischions et la longueur du bassin.

Tableau 3 : Moyennes et Écart-type des paramètres quantitatifs de la tête

\begin{tabular}{|c|c|c|c|c|c|c|c|c|c|c|c|c|c|}
\hline Sexe & & $\mathrm{N}$ & LT & $\mathrm{LCr}$ & LF & It & Icr & LO & $\mathrm{CM}$ & Lco & DPP & DBB & $\mathrm{CC}$ \\
\hline \multirow[t]{2}{*}{ Mâle } & Moyenne & 36 & $55,69^{a c}$ & $21,42^{\mathrm{ac}}$ & $34,28^{a c}$ & $26,96^{\mathrm{ac}}$ & $29,39^{a c}$ & $19,69 \mathrm{abc}$ & $44,60^{\mathrm{ac}}$ & $61,67^{a b}$ & $75,57^{a b c}$ & $22,74^{\mathrm{ac}}$ & $40,17^{\mathrm{ac}}$ \\
\hline & Écart-type & & 3,99 & 2,75 & 3,87 & 3,87 & 3,70 & 1,23 & 3,96 & 16,29 & 19,53 & 3,18 & 7,87 \\
\hline \multirow[t]{2}{*}{ Femelle } & Moyenne & 423 & $51,81^{b}$ & $20,21^{b}$ & $31,61^{b}$ & $22,72^{b}$ & $23,21^{b}$ & 19,39 ba & $41,56^{b}$ & $63,07^{\text {ba }}$ & $67,50^{\mathrm{bac}}$ & $20,78^{b}$ & $30,77^{b}$ \\
\hline & Écart-type & & 3,81 & 2,42 & 3,57 & 2,00 & 2,48 & 1,77 & 2,78 & 18,44 & 25,13 & 5,15 & 9,09 \\
\hline \multirow[t]{2}{*}{ Castré } & Moyenne & 16 & $57,31^{\text {ca }}$ & $22,63^{c a}$ & 34,69 ca & $26,06^{\text {ca }}$ & $27,13^{\mathrm{ca}}$ & $20,38^{\text {ca }}$ & 44,69 ca & $79,91^{c}$ & $63,94^{\text {cab }}$ & $23,31^{\text {ca }}$ & 41,91 ca \\
\hline & Écart-type & & 5,04 & 2,31 & 4,45 & 3,40 & 2,45 & 1,46 & 2,89 & 16,15 & 20,81 & 3,28 & 6,87 \\
\hline \multirow[t]{2}{*}{ Race } & Moyenne & 475 & 52,30 & 20,38 & 32,05 & 23,15 & 23,81 & 19,44 & 41,91 & 64,49 & 68,94 & 21,40 & 32,33 \\
\hline & Écart-type & & 4,10 & 2,49 & 4,49 & 2,56 & 3,12 & 1,73 & 3,02 & 16,84 & 23,46 & 4,10 & 8,67 \\
\hline
\end{tabular}

$\mathrm{N}$ : taille de l'échantillon ; LT : longueur de la tête ; LCr : longueur du crane ; LF : longueur de la face ; It : largeur de la tête ; Icr : largeur du crâne ; LO : longueur de l'oreille ; CM : circonférence du museau ; DPP : Distance Pointe-Pointe corne ; DBB : distance base-base corne ; CC : circonférence de la corne.

NB : Dans la même colonne, deux moyennes portant des lettres différentes $(a, b$, ou $c)$ sont statistiquement différentes $(P<0,05)$.

Tableau 4 : Matrice de corrélation entre les paramètres de la tête

\begin{tabular}{|c|c|c|c|c|c|c|c|c|c|c|c|c|c|c|c|c|}
\hline Variables & $\mathrm{LT}$ & $\mathrm{Lt}$ & $\mathrm{LCr}$ & $\mathrm{LF}$ & $\mathrm{Icr}$ & $\mathrm{CM}$ & Lco & DPP & DBB & $\mathrm{CC}$ & LO & $\mathrm{P}$ & Age & $C$ & $\mathrm{~F}$ & $M$ \\
\hline LT & 1,00 & & & & & & & & & & & & & & & \\
\hline It & 0,61 & 1,00 & & & & & & & & & & & & & & \\
\hline $\mathrm{LCr}$ & 0,52 & 0,17 & 1,00 & & & & & & & & & & & & & \\
\hline LF & 0,78 & 0,58 & $-0,13$ & 1,00 & & & & & & & & & & & & \\
\hline Icr & 0,45 & 0,48 & 0,37 & 0,24 & 1,00 & & & & & & & & & & & \\
\hline CM & 0,63 & 0,52 & 0,41 & 0,44 & 0,40 & 1,00 & & & & & & & & & & \\
\hline Lco & 0,46 & 0,31 & 0,49 & 0,18 & 0,36 & 0,47 & 1,00 & & & & & & & & & \\
\hline DPP & 0,22 & 0,17 & 0,07 & 0,20 & 0,14 & 0,24 & 0,36 & 1,00 & & & & & & & & \\
\hline DBB & 0,00 & 0,04 & 0,12 & $-0,09$ & 0,25 & 0,07 & 0,18 & 0,33 & 1,00 & & & & & & & \\
\hline CC & 0,46 & 0,43 & 0,33 & 0,30 & 0,56 & 0,39 & 0,57 & 0,27 & 0,34 & 1,00 & & & & & & \\
\hline LO & 0,42 & 0,22 & 0,26 & 0,30 & 0,18 & 0,32 & 0,17 & 0,09 & 0,03 & 0,11 & 1,00 & & & & & \\
\hline$P$ & 0,22 & 0,15 & 0,18 & 0,12 & 0,22 & 0,21 & 0,21 & 0,13 & 0,06 & 0,24 & 0,15 & 1,00 & & & & \\
\hline Age & 0,41 & 0,23 & 0,17 & 0,35 & 0,02 & 0,45 & 0,43 & 0,18 & $-0,14$ & 0,02 & 0,25 & 0,06 & 1,00 & & & \\
\hline C & 0,21 & 0,27 & 0,16 & 0,13 & 0,33 & 0,21 & 0,20 & $-0,10$ & 0,11 & 0,32 & 0,10 & 0,10 & $-0,04$ & 1,00 & & \\
\hline $\mathrm{F}$ & $-0,25$ & $-0,39$ & $-0,20$ & $-0,15$ & $-0,53$ & $-0,23$ & $-0,14$ & 0,15 & $-0,07$ & $-0,31$ & $-0,12$ & $-0,08$ & 0,11 & $-0,74$ & 1,00 & \\
\hline M & 0,13 & 0,27 & 0,11 & 0,07 & 0,41 & 0,09 & $-0,01$ & $-0,11$ & $-0,02$ & 0,11 & 0,06 & 0,00 & $-0,12$ & $-0,04$ & $-0,65$ & 1,00 \\
\hline
\end{tabular}


Tableau 5 : Les paramètres qualitatifs du corps et de la tête

\begin{tabular}{|c|c|c|c|c|c|c|c|c|c|}
\hline \multirow{2}{*}{ Variables } & \multirow[t]{2}{*}{ Modalités } & \multicolumn{2}{|c|}{ Mâles } & \multicolumn{2}{|c|}{ Femelles } & \multicolumn{2}{|c|}{ Castrés } & \multicolumn{2}{|c|}{ Race } \\
\hline & & $\mathbf{N}$ & $\%$ & $\mathbf{N}$ & $\%$ & $\mathbf{N}$ & $\%$ & $\mathrm{~N}$ & $\%$ \\
\hline \multirow[t]{2}{*}{ Profil de la tête } & Rectiligne & 26 & 68,42 & 368 & 88,25 & 13 & 84,21 & 407 & 85,86 \\
\hline & Convexe & 12 & 31,58 & 49 & 11,75 & 6 & 15,79 & 67 & 14,14 \\
\hline \multirow[t]{2}{*}{ Orientation de l'oreille } & Dressée & 0 & 0,00 & 0 & 0,00 & 1 & 5,26 & 1 & 0,21 \\
\hline & Latérale & 38 & 100,00 & 417 & 100,00 & 18 & 94,74 & 473 & 99,79 \\
\hline Forme de l'oreille & Arrondie & 38 & 100,00 & 417 & 100 & 19 & 100,00 & 474 & 100,00 \\
\hline \multirow[t]{2}{*}{ Présence des cornes } & Oui & 38 & 100,00 & 407 & 97,60 & 19 & 100,00 & 464 & 97,89 \\
\hline & Non & 0 & 0,00 & 10 & 2,40 & 0 & 0,00 & 10 & 2,11 \\
\hline \multirow[t]{3}{*}{ Couleur des cornes } & Noir & 2 & 5,26 & 17 & 4,18 & 0 & 0,00 & 19 & 4,09 \\
\hline & Blanc & 35 & 92,11 & 321 & 78,87 & 15 & 78,95 & 371 & 79,96 \\
\hline & Brun & 1 & 2,63 & 69 & 16,95 & 4 & 21,05 & 74 & 15,95 \\
\hline \multirow{2}{*}{ Attache des cornes } & Flottante & 1 & 2,63 & 11 & 2,70 & 0 & 0,00 & 12 & 2,59 \\
\hline & Fixée & 37 & 97,37 & 396 & 97,30 & 19 & 100,00 & 452 & 97,41 \\
\hline \multirow[t]{5}{*}{ Orientation des cornes } & Latérale & 4 & 10,53 & 18 & 4,43 & 1 & 5,26 & 23 & 4,97 \\
\hline & Vers le haut & 26 & 68,42 & 205 & 50,50 & 14 & 73,69 & 245 & 52,92 \\
\hline & Vers le bas & 0 & 0,00 & 7 & 1,72 & 0 & 0,00 & 7 & 1,51 \\
\hline & En avant & 5 & 13,16 & 76 & 18,72 & 1 & 5,26 & 82 & 17,71 \\
\hline & En arrière & 3 & 7,89 & 100 & 24,63 & 3 & 15,79 & 106 & 22,89 \\
\hline \multirow[t]{3}{*}{ Appréciation de la ligne du dos } & Rectiligne & 27 & 71,05 & 371 & 88,97 & 12 & 63,16 & 410 & 86,50 \\
\hline & Concave & 0 & 0,00 & 3 & 0,72 & 1 & 5,26 & 4 & 0,84 \\
\hline & Convexe & 11 & 28,85 & 43 & 10,31 & 6 & 31,58 & 60 & 12,66 \\
\hline Profil de la croupe & Incliné & 38 & 100,00 & 417 & 100,00 & 19 & 100,00 & 474 & 100,00 \\
\hline Longueur de la queue & Longue & 38 & 100,00 & 417 & 100,00 & 19 & 100,00 & 474 & 100,00 \\
\hline
\end{tabular}

Traits quantitatifs au niveau de la tête

Valeurs moyennes des paramètres quantitatifs de la

tête : Le tableau 3 donne les moyennes des paramètres quantitatifs de la tête avec les résultats de leur comparaison selon le sexe. Le tableau fait ressortir des différences significatives entre les moyennes des groupes deux à deux. Ainsi, pour la longueur de la tête $\left(L T_{M}=L T_{C}\right.$, $\left.L T_{C}>L T_{F}\right)$, il en est de même pour la longueur du crane $(\mathrm{LCr})$, la longueur de la face (LF), la largeur de la tête (It), la largeur du crâne (Icr), la circonférence du museau $(\mathrm{CM})$, la distance base-base corne (DBB), et la circonférence de la corne (CC). II existe donc des différences significatives entre les mâles et femelles d'une part et d'autre part entre les castrés et les femelles. On peut relever que pour ces paramètres, la différence n'est pas significative entre les mâles entiers et les mâles castrés. On enregistre pour la longueur de l'oreille (LO), une différence significative entre femelle et castré. II existe également pour la longueur de la corne, une différence significative entre mâle et castré d'une part et entre femelle et castré d'autre part. 


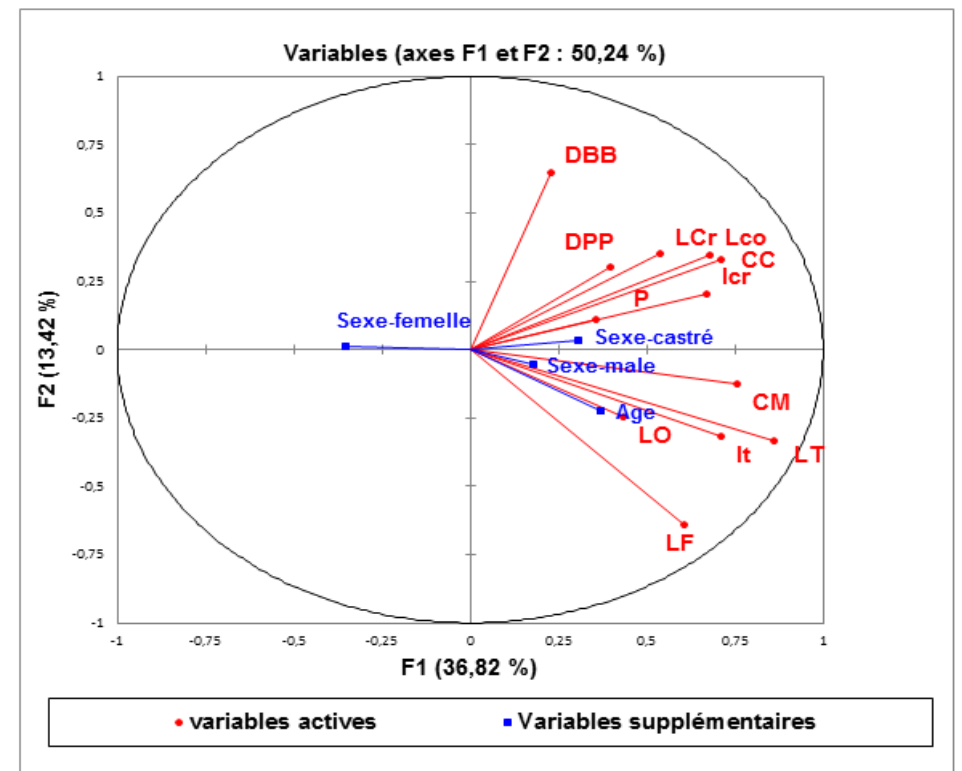

Figure 5 : Cercle de corrélation des paramètres quantitatifs de la tête

LT : longueur de la tête ; It : largeur de la tête ; LCr : Longueur du crane ; LF : longueur de la face ; Icr : largeur du crâne ;

$\mathrm{CM}$ : circonférence du museau ; LCo : longueur corne; DPP : distance pointe-pointe corne; DBB : distance base-base corne ; CC : circonférence de la corne ; LO : longueur de l'oreille ; P : poids.

Corrélations entre les paramètres quantitatifs de la tête : Le tableau 4 donne les coefficients de corrélation entre les différents paramètres de la tête en composantes principales et aussi celles de l'âge et du sexe en composantes supplémentaires. L'analyse du tableau met en évidence les corrélations entre les paramètres de la tête et ces corrélations varient de corrélations moyennes positives et négatives (coefficient de corrélation compris entre 0,5 à 0,7 et $-0,5$ à $-0,7)$ à corrélations faibles positives et négatives (coefficient de corrélation appartenant à l'intervalle]-0,5; 0,5[).

Les corrélations moyennes sont enregistrées : entre $L T$ et $\operatorname{LF}(0,78)$; entre LT et It $(0,61)$; entre CM et LT $(0,632)$. Les corrélations faibles apparaissent entre $\mathrm{LT}$ et $\mathrm{Icr}$ $(0,45)$; entre DBB et LT $(0,001)$.

La figure 5 donne la représentation dans le plan factoriel $\left(F_{1} X F_{2}\right)$ des paramètres quantitatifs de la tête en rouge et les paramètres supplémentaires en bleu.

$F_{1}$ et $F_{2}$ ont les valeurs propres les plus élevées et expliquent $50,24 \%$ des observations. Tous les paramètres sont bien représentés sur ces axes. Cependant on remarque qu'à l'exception de DBB qui est mieux représenté sur l'axe $F_{2}$ et LF qui est bien représenté sur les deux axes, tous les autres paramètres de la tête sont mieux représentés sur l'axe $F_{1}$. DBB a une corrélation presque nulle avec les autres paramètres $y$ compris les paramètres supplémentaires comme le sexe. On déduit alors que les autres paramètres sont caractéristiques de la race, car ils sont bien corrélés positivement avec les mâles et les castrés et négativement avec les femelles.

Paramètres qualitatifs du corps et de la tête: Le tableau 5 donne les paramètres qualitatifs du corps et de la tête en fonction du sexe et également ceux de la race. II ressort de l'analyse de ce tableau que pour le profil de la tête, deux types de profil sont observés: le profil rectiligne et le profil convexe. Le profil rectiligne est dominant chez tous les sexes et même au sein de la race, comme en témoignent les pourcentages de $68,42 \%$, $88,25 \%, 84,21 \%$ et $85,86 \%$ respectivement chez les mâles, les femelles, les castrés et au sein de la race.

Concernant l'orientation de l'oreille, deux types se distinguent: dressé et latérale. Chez les mâles et les femelles, $100 \%$ des animaux échantillonnés contre $94,74 \%$ chez les castrés, ont les oreilles orientées latéralement. Ce taux est de $99,79 \%$ au sein de la race. Tous les animaux ont les oreilles arrondies. 
Tableau 6 : Coloration et pigmentation des différentes parties du corps et de la tête

\begin{tabular}{|c|c|c|c|c|c|c|c|c|c|}
\hline \multirow[b]{2}{*}{ Variables } & \multirow[t]{2}{*}{ Modalités } & \multicolumn{2}{|c|}{ Mâle } & \multicolumn{2}{|c|}{ Femelle } & \multicolumn{2}{|c|}{ Castré } & \multicolumn{2}{|c|}{ Race } \\
\hline & & $\mathbf{N}$ & $\%$ & $\mathbf{N}$ & $\%$ & $\mathbf{N}$ & $\%$ & $\mathbf{N}$ & $\%$ \\
\hline \multirow[t]{10}{*}{ Couleur de la tête } & Noire & 1 & 2,60 & 11 & 2,60 & 1 & 5,30 & 13 & 2,74 \\
\hline & Blanche & 27 & 71,10 & 274 & 65,70 & 11 & 57,90 & 312 & 65,82 \\
\hline & Rouge & 5 & 13,20 & 96 & 23,00 & 6 & 31,60 & 107 & 22,57 \\
\hline & Rouan & 0 & 0,00 & 2 & 0,50 & 0 & 0,00 & 2 & 0,42 \\
\hline & Sable & 4 & 10,50 & 4 & 1,00 & 0 & 0,00 & 8 & 1,69 \\
\hline & Fauve & 0 & 0,00 & 12 & 2,90 & 0 & 0,00 & 12 & 2,53 \\
\hline & Cendre & 0 & 0,00 & 1 & 0,20 & 0 & 0,00 & 1 & 0,21 \\
\hline & Brune & 1 & 2,60 & 11 & 2,60 & 1 & 5,30 & 13 & 2,74 \\
\hline & Froment & 0 & 0,00 & 5 & 1,20 & 0 & 0,00 & 5 & 1,05 \\
\hline & Grise & 0 & 0,00 & 1 & 0,20 & 0 & 0,00 & 1 & 0,21 \\
\hline \multirow[t]{2}{*}{ Couleur du mufle } & Pigmentée & 6 & 15,80 & 51 & 12,20 & 2 & 10,50 & 59 & 12,45 \\
\hline & Non pigmentée & 32 & 84,20 & 366 & 87,80 & 17 & 89,50 & 415 & 87,55 \\
\hline \multirow[t]{13}{*}{ Couleur de la robe } & Blanc & 23 & 60,50 & 210 & 50,40 & 8 & 42,10 & 241 & 50,84 \\
\hline & Rouge foncé & 0 & 0,00 & 6 & 1,40 & 0 & 0,00 & 6 & 1,27 \\
\hline & Rouge clair & 2 & 5,30 & 50 & 12,00 & 4 & 21,10 & 56 & 11,81 \\
\hline & Fauve & 1 & 2,60 & 48 & 11,50 & 3 & 15,80 & 52 & 10,97 \\
\hline & Gris & 5 & 13,20 & 42 & 10,10 & 1 & 5,30 & 48 & 10,13 \\
\hline & Noir & 0 & 0,00 & 2 & 0,50 & 0 & 0,00 & 2 & 0,42 \\
\hline & Sable & 6 & 15,80 & 35 & 8,40 & 1 & 5,30 & 42 & 8,86 \\
\hline & Froment & 0 & 0,00 & 14 & 3,40 & 0 & 0,00 & 14 & 2,95 \\
\hline & Pie rouge & 1 & 2,60 & 4 & 1,00 & 2 & 10,50 & 7 & 1,48 \\
\hline & Rouan & 0 & 0,00 & 2 & 0,50 & 0 & 0,00 & 2 & 0,42 \\
\hline & Charbonnée & 0 & 0,00 & 1 & 0,20 & 0 & 0,00 & 1 & 0,21 \\
\hline & Bringée & 0 & 0,00 & 2 & 0,50 & 0 & 0,00 & 2 & 0,42 \\
\hline & Brune & 0 & 0,00 & 1 & 0,50 & 0 & 0,00 & 1 & 0,21 \\
\hline \multirow[t]{2}{*}{ Pigmentation de la peau } & Pigmentée & 23 & 60,50 & 304 & 72,90 & 16 & 84,20 & 343 & 72,36 \\
\hline & Non Pigmentée & 15 & 39,50 & 113 & 27,10 & 3 & 15,80 & 131 & 27,64 \\
\hline \multirow[t]{2}{*}{ Couleur du sabot } & Pigmentée & 20 & 52,60 & 259 & 62,10 & 12 & 63,20 & 291 & 61,39 \\
\hline & Non pigmentée & 18 & 47,40 & 158 & 37,90 & 7 & 36,80 & 183 & 38,61 \\
\hline \multirow[t]{2}{*}{ Couleur des paupières } & Pigmentée & 19 & 50,00 & 242 & 58,00 & 14 & 73,70 & 275 & 58,02 \\
\hline & Non pigmentée & 19 & 50,00 & 175 & 42,00 & 5 & 26,30 & 199 & 41,98 \\
\hline
\end{tabular}

Chez $100 \%$ des mâles et des castrés, contre $97,60 \%$ chez les femelles, on note la présence de cornes. Les cornes sont soit fixées, ou flottante. La totalité (100\%) des castrés contre $97,37 \%$ des mâles et $97,30 \%$ des femelles ont les cornes fixées. L'orientation des cornes varie de latérale, vers le haut, vers le bas, en avant ou en arrière. On note au sein de la race que, $52,92 \%$ des animaux ont une orientation des cornes vers le haut. Les cornes sont majoritairement de couleur blanche dans les 3 catégories et sur l'ensemble de l'échantillon $(79,96 \%)$. Trois types d'appréciation de la ligne du dos se distinguent : il s'agit de rectiligne, concave et convexe. Sur l'ensemble de l'échantillon on enregistre $86,50 \%$ des animaux à dos rectiligne ; $12,66 \%$ à dos convexe et enfin $0,84 \%$ à dos concave. Le profil de la croupe est incliné et la queue est longue pour l'ensemble de l'échantillon examiné. Le tableau 6 donne les colorations et pigmentations des différentes parties du corps et de la tête. L'analyse du tableau fait ressortir dix couleurs pour la tête. Ce sont le noir, blanc, rouge, rouan, sable, fauve, cendre, brun, froment, et gris. Les pourcentages d'apparition de ces couleurs dans notre échantillon varient de $0,21 \%$ (cendre, gris) à $65,82 \%$ (blanc) avec $22,57 \%$ pour le rouge. Les couleurs de robe enregistrées vont du blanc au noir avec $50,40 \%$ de femelles de robe blanche contre $60,50 \%$ chez les mâles entiers. De façon générale, la robe est la robe dominante chez la race Kouri (50,84\% des individus de l'échantillon). La robe la moins représentée est la robe charbonnée $(0,218)$. La peau, les sabots, les paupières sont pigmentées pour la majeure partie des animaux. Par contre le mufle n'est pas pigmenté chez $87,55 \%$ des animaux de l'échantillon. 


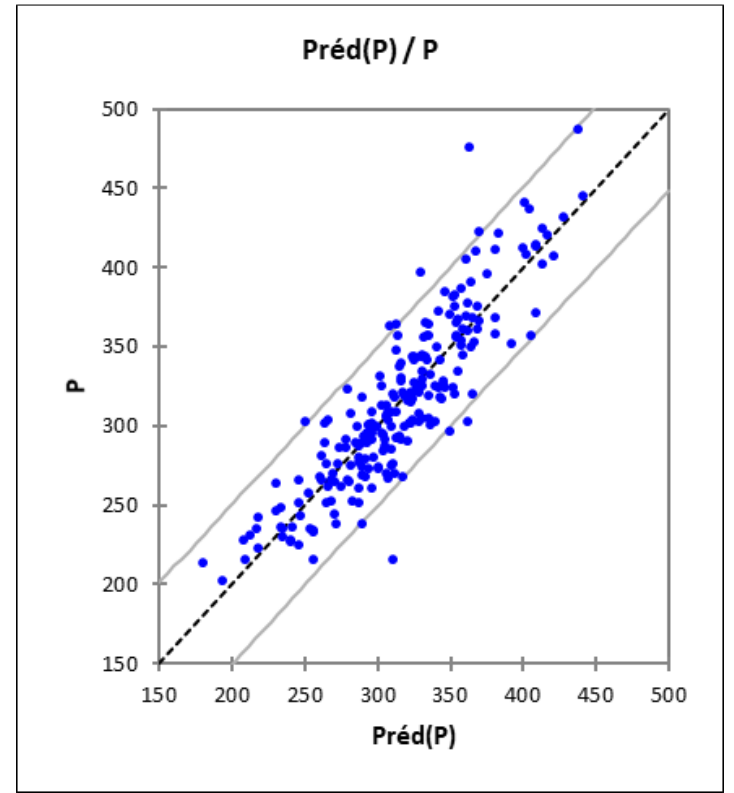

Figure 6 : Droite de régression du poids en fonction de LC, HG, PT, HS, Lsi et Pp

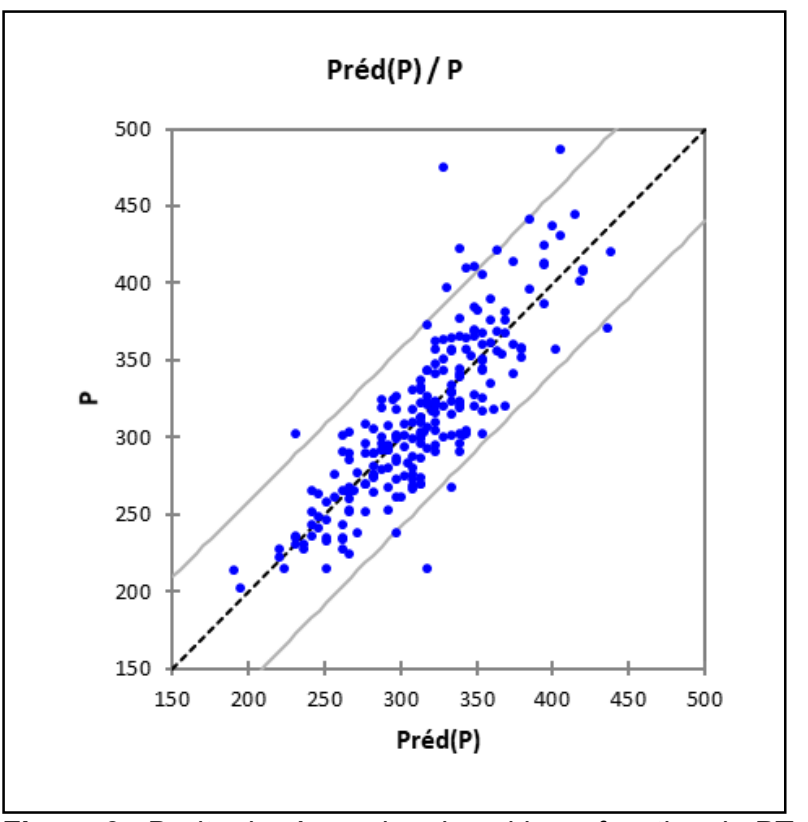

Figure 8 : Droite de régression du poids en fonction du PT

Formule barymétrique applicable au taurin Kouri du Niger : Une première régression qui a pris en compte les six paramètres quantitatifs à savoir le périmètre thoracique (PT), la hauteur au garrot $(\mathrm{HG})$, la hauteur au sacrum (HS), la longueur scapulo-ischiale (Lsi), la

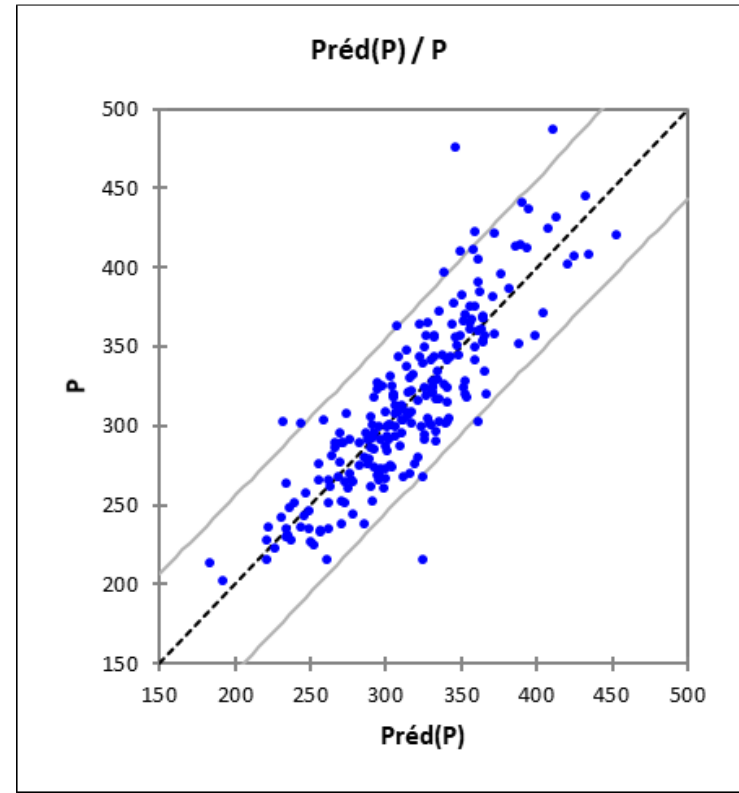

Figure7 : Droite de régression du poids en fonction de PT, $H G$ et $H S$ longueur du corps (LC) et la profondeur poitrine (Pp) selon la formule :

$\mathrm{P}=\quad$ - $643,61+2,02^{*} \mathrm{LC}+0,97^{*} \mathrm{HG}+3,44^{*} \mathrm{PT}$

$0,23^{*} \mathrm{HS}+0,56^{*} \mathrm{Lsi}+0,67^{*} \mathrm{Pp}$;

Donne le coefficient d'ajustement suivant $R_{1}{ }^{2}=0,79$. 
La figure 6 donne la dispersion des individus en fonction de la droite de régression.

Dans la deuxième régression, où seuls trois paramètres corporels ont été utilisés (PT, $\mathrm{HG}$ et $\mathrm{HS}$ ), avec la formule $P=-598,37+2,39^{*} \mathrm{HG}+3,99^{*} \mathrm{PT}-0,07^{*} \mathrm{HS}$; le coefficient d'ajustement est $R_{2}{ }^{2}=0,74$.

La figure 7 donne la dispersion en fonction de la droite de régression.

\section{DISCUSSION}

Paramètres morpho biométriques du taurin Kouri et leurs corrélations : Les valeurs de la hauteur au garrot $(125,65 \pm 6,08)$, de la longueur des cornes $(64,49 \pm 16,84)$ et de la circonférence des cornes $(32,33 \pm 8,67)$ trouvées dans notre étude appartiennent aux intervalles respectifs de 140-150 cm, 70-130 cm et 20-55 cm donnés par Joshi et Ralph (1957) à travers une étude menée au Tchad sur la race Kouri. Cependant, le poids obtenu $(314,38 \pm 54,36)$, ainsi que la circonférence des cornes $(32,33 \pm 8,67)$ sont inférieurs à ceux rapportées dans Niger (2003) et Tchad (2003) chez la race Kouri. Malbrant et al., (1947) ont rapporté chez le mâle, la femelle et le castré Kouri élevés au Tchad, des valeurs supérieures à celles obtenues dans notre étude pour le poids, la hauteur au sacrum, la hauteur au garrot, le périmètre thoracique et la longueur de la tête. Cependant ces mêmes auteurs ont trouvé des valeurs inférieures, pour la longueur scapulo-ischiale et des résultats semblables pour la largeur aux hanches et celle de la tête au niveau de tous les sexes. Koné (1948) a rapporté pour la race Kouri élevée dans la zone de N'Guigmi, des valeurs supérieures à celles trouvées dans notre étude pour le poids, la hauteur au sacrum, la hauteur au garrot, la longueur scapulo-ischiale, le périmètre thoracique et la longueur de la tête. La valeur de la largeur aux hanches trouvée par cet auteur est inférieure à celle de notre étude qui est de $46,44 \pm 4,074 \mathrm{~cm}$. On remarque par ailleurs, que pour la majorité des paramètres rapportés par Malbrant et al., (1947), les valeurs obtenues chez le castré sont supérieures à celles obtenues chez le mâle, qui sont elles aussi supérieures à celles trouvées chez la femelle. Cette observation semble être la même dans notre étude. En effet à l'exception de la largeur aux ischions, la longueur du corps et la largeur de la tête, les valeurs trouvées chez le castré sont supérieures à celles du mâle, qui sont à leur tour supérieures à celles de la femelle sauf pour la longueur du bassin et la longueur des cornes. Ces résultats laissent penser que la castration pourrait présenter des effets positifs sur le développement des différentes parties du corps. Les
Pour la troisième régression linéaire qui n'a pris en compte que le PT, et dont la formule utilisée est : $P=$ $485,53+5,12^{\star} \mathrm{PT} ; \mathrm{R}_{3}{ }^{2}=0,71$.

La figure 8 donne la dispersion en fonction de la droite de régression.

L'analyse montre que $R_{1}{ }^{2}>R_{2}{ }^{2}>R_{3}{ }^{2}$, ce qui signifie que meilleure la régression est celle qui combine les six paramètres (HG, $P T, H S$, LC, Lsi et Pp), $R_{1}{ }^{2}=0,79$.

similitudes et les différences enregistrées pour certains paramètres au sein de la race Kouri dans notre étude et aussi en comparaison avec les autres études, pourraient s'expliquer à travers les conditions climatiques, le génotype de l'animal ou bien par les pratiques d'élevage notamment la forte introgression de la race Kouri par les zébus Bororo, Arabe et Goudali, du fait des croisements. La comparaison de nos résultats avec ceux de quelques études effectuées sur les races taurines africaines et zébus du Niger, a également révélé des similitudes et des différences. C'est ainsi que pour les races taurines Baoulé, N'Dama et Kapsiki, Doutressole (1947), cité par Kaboré (2012), Joshi et Ralph, (1957) et Dineur et Thys (1986), ont trouvé pour la hauteur au garrot, le poids, la longueur scapulo-ischiale et le périmètre thoracique, des valeurs inférieures à celles trouvées dans notre étude. Les différences entre nos résultats et ceux de ces auteurs laissent apparaître que la race Kouri représente l'une des plus grandes races taurines d'Afrique. Les valeurs de la hauteur au garrot et du poids chez le mâle et la femelle Kouri, obtenues au cours de cette étude sont semblables à celles des zébus Azawak et Djelli, mais inferieures à celle des Bororo et Goudali rapportés dans Niger (2003). La longueur moyenne des cornes $(64,49 \pm 16,84 \mathrm{~cm})$ trouvée dans notre étude est inférieure à celle trouvée chez la race Bororo (Niger, 2003). Chez l'Azawak, pour la longueur de l'oreille, le résultat rapporté par Joshi et Ralph (1957) est supérieur à celui trouvé dans notre étude qui est de 19,44 $\pm 1,73 \mathrm{~cm}$. II ressort de ces comparaisons que du point de vue de la taille, la race Kouri est comparable aux zébus Azawak et Djelli, tandis qu'elle est légèrement plus petite que la Bororo et la Goudali. Le profil de la tête et la couleur de la robe rapportés dans Niger (2003) et Tchad (2003) correspondent à ceux trouvés majoritairement dans notre étude, c'est-à-dire $85,86 \%$ pour le profil rectiligne et $50,84 \%$ pour la couleur blanche claire de la robe. Les caractéristiques des cornes, l'appréciation de la ligne du dos et le profil de la croupe chez la race Kouri, rapportés par Joshi et Ralph (1957) ; Queval et al., (1971) et Tchad 
(2003), sont comparables à celles trouvées dans notre échantillon. Nos résultats sur les caractéristiques de la queue, sont conformes à ceux trouvés par Zangui en 1986 sur la Kouri au Niger. En somme, pour tous les paramètres qualitatifs étudiés, on remarque que les résultats sont semblables à ceux trouvés par d'autres auteurs. Ces résultats laissent penser que les paramètres qualitatifs dépendent exclusivement du génotype de la race indépendamment des conditions climatiques et d'élevage.

Formule barymétrique applicable au taurin Kouri du Niger : La connaissance du poids vif chez les bovins est d'un intérêt qui n'est plus à démontrer. En effet certaines interventions sur les animaux (traitement, vaccination, déparasitage, rationnement, etc.) nécessitent la connaissance de leur poids. Or l'absence de bascule dans la plupart des élevages rend cette détermination très imprécise tant pour l'éleveur que pour le technicien sur le terrain. Aussi l'utilisation de la bascule nécessite le plus souvent des moyens importants tant financièrement que matériellement. Alors qu'une technique simple, la barymétrie, permet d'obtenir des estimations satisfaisantes du poids vif à partir des relations entre

\section{CONCLUSION}

L'amélioration de la productivité animale passe inévitablement par la connaissance des races locales. II est donc nécessaire de bien connaitre les caractéristiques physiques de nos races afin de pouvoir les comparer à d'autres. Les résultats obtenus, montrent que la race Kouri présente une hétérogénéité sur le plan phénotypique comme en témoigne la diversité de couleurs de la robe et de formes des cornes. Cependant, l'absence de la bosse peut être caractéristique de cette race au Niger. Cette étude a fait ressortir des résultats intéressants. En effet ces résultats montrent que, la Kouri est l'une des plus grandes races taurines d'Afrique par rapport à la comparaison de certaines mensurations corporelles comme la hauteur au garrot, le périmètre thoracique, la hauteur au sacrum, la longueur du corps, la longueur scapulo-ischiale et le poids. L'étude montre

\section{RÉFÉRENCES BIBLIOGRAPHIQUES}

APESS, 2014. Éléments de bilan du soutien public à l'élevage au Niger depuis Maputo. Document de travail, 12 pages.

Bourzat D., Idriss A., ZEUH V., 1992. La race Kouri une population bovine en danger d'absorption. Anim. Genet. Ressource Information 9, 13-21, FAO, Rome. diverses mensurations corporelles et le poids vif de bovins (Bulgden et al., 1984). La prise en compte de certains paramètres (hauteur au garrot, hauteur au sacrum, périmètre thoracique, longueur scapulo-ischiale, longueur du corps et profondeur de la poitrine), dans l'établissement des formules barymétriques a été rapportée par plusieurs auteurs (Delage et al., 1955 ; Pagot et Delaine, 1959 ; Chollou et al., 1978 ; Poivey et al., 1980 ; Dineur et Thys, 1986 ; Dodo et al., 2001). Par ailleurs, tous ces auteurs sont unanimes que, pour une utilisation individuelle optimale de ces paramètres, le périmètre thoracique serait le mieux indiqué. Ces résultats corroborent ceux de notre étude car le périmètre thoracique, présente le coefficient de corrélation le plus élevé avec le poids $(0,843)$. Aussi la formule pratique est celle avec le PT car un ruban peut être facilement élaboré tandis que la mensuration de 6 paramètres et leur inclusion dans la formule de calcul sont très fastidieuses. Néanmoins le modèle de régression établie à partir de 6 paramètres, (HG, HS, Lsi, PT, LC, Pp) donne le coefficient d'ajustement le plus intéressant, par conséquent les valeurs du poids les plus proches de la réalité.

également que du point de vue de la taille, la race Kouri est comparable à certaines races de zébus nigériens comme l'Azawak et la Djelli, et aussi qu'elle est très proche de la Bororo et de la Goudali. Aussi cette étude a permis de mettre en évidence des relations entre quelques paramètres quantitatifs et le poids. $\mathrm{A}$ travers ces relations, des formules barymétriques ont été proposées. L'application de ces formules barymétriques pour l'estimation de la valeur financière des animaux et le dosage des médicaments sur le terrain permettra d'atténuer les problèmes de l'utilisation des bascules qui sont très couteuses et difficiles à transporter. Cette étude pose les jalons de la connaissance de la race taurine Kouri au Niger pour une amélioration génétique et une base pour une gestion de cette ressource.

Buldgen A., Compere R. et Riboux A., 1984. Recherche d'une formule barymétrique adaptée aux bovins de type Djakoré des élevages villageois du Sénégal oriental. Tropicultura 2(3) : 86-90.

Christian L., Ousseini S. et Marc L., 1998. Évolution des ressources en eau dans le département de Diffa 
(bassin du lac Tchad, sud-est nigérien). IAHS Publ.n²52 : 281-288.

Chollou M., Denis J.P. et Gauchet D., 1978. Calcul d'une formule barymétrique adaptée au zébu Gobra. Rév. Elev. Méd. Vét. Pays trop. 31(4) : 447-450.

Delage J., Poly J. et Vissac B., 1955. Étude de l'efficacité relative de diverses formules de barymétrie applicables aux bovins. Ann - zootech $3: 219$ 231.

Dineur B. et Thys E., 1986. Les Kapsiki : race taurine de l'extrême Nord camerounais. I. Introduction et barymétrie. Rev. Elev. Méd. Vét. Pays trop. 39 : 435-442.

Dodo K., Pandey V.S. et Illiassou M.S., 2001. Utilisation de la barymétrie pour l'estimation du poids chez le zébu Azawak au Niger. Rev. Elev. Méd. Vét Pays trop. 54 (1) : 63-68.

Doutressole G., 1947. L'élevage en Afrique Occidentale Française. Edition Larose, Paris, 298 p.

FAO., 2003a. Dynamique des populations, disponibilités en terres et adaptation des régimes fonciers : le cas du Niger. Edition Francis GENDREAU, Mumpasi LUTUTALA.

FAO., 2003b. La situation de la communication pour le développement au Niger (État des lieux) Tome 1 Rome, $171 \mathrm{p}$.

FAO., 2013. Commission des ressources génétiques pour l'Alimentation et l'Agriculture. Caractérisation phénotypique des ressources génétiques animales. Directives FAO sur la production et la santé animales $n^{\circ} 11$. Rome, $151 \mathrm{p}$.

INS., 2015. Monographie sur les adolescents : Région de Diffa, $76 \mathrm{p}$.

INS., 2014. Le Niger en chiffre 2014, $84 p$

Joshi R et Ralph W., 1957. Les bovins d'Afrique : types et races. FAO, Rome, $317 p$

Kaboré M., 2012.Étude de la diversité génétique des taurins Baoulé du Burkina Faso à l'aide de marqueurs microsatellites. Mémoire de Diplôme d'Études Approfondies de l'Université de Ouagadougou, $68 \mathrm{p}$

Koné K., 1948. Le bœuf du Lac Tchad de la région de N'Guigmi. Bull. serv. Elev. Ind. Antm. AOF, 1 (2) : 47-65.

Malbrant R., Receveur P. et Sabrin R., 1947. Le bœuf du Lac Tchad. Rev. Elev. Méd. Vét. Pays trop. 1(1) : 37-42; 1(2) : 109-129.

Marichatou H., Nervo T., Semita C., Issa M., Abdou M., 2011. Évaluation des paramètres reproductifs et zootechniques de la race taurine Kouri au Niger (résumé d'une communication). In : 6e Colloque international « TURIN SAHEL » : Contribution des savoirs endogènes au développement de la santé humaine, animale et à la conservation de la biodiversité, 28 février-02 mars 2011, Abomey Calavi. Bénin. 72p.

Mian O.K., 2008. Dynamique des changements dans le secteur de l'élevage au Tchad: le cas de la filière laitière de N'Djamena. Thèse de doctorat d'État Agro Paris Tech, $239 \mathrm{p}$.

Niger, Ministère de l'élevage, 2013. Stratégie de Développement Durable de L'élevage (SDDEL 2013-2035),76 p.

Niger, SOW - AnGR., 2003. État des ressources génétiques animales dans le monde. Rapport national. Niger, Niamey INRA,104p.

Pagot J. et Delaine R., 1959. Étude biométrique de la croissance des taurins N'Dama. Rév. Elev. Méd. Vét. Pays trop. $4: 405-416$.

Poivey J.P., Landais E. et Seitz J.L., 1980. Utilisation de la barymétrie chez les races taurines locales de la Côte d'Ivoire. Rév. Elev. Méd. Vét. Pays trop. 33 (3) : 311-317.

Queval R., Petit J.P., Tacher G., Provost A., Pagot J., 1971. Le Kouri : race bovine du lac Tchad: Introduction générale à son étude zootechnique et biochimique : origine et écologie de la race. Rév. Elev. Méd. Vét. Pays trop. 24 (4) : 667-687.

Queval R. et Petit J.P., 1973. Le Kouri : race bovine du Lac Tchad. Rév. Elev. Méd. Vét. Pays trop. 26 : 235-244.

Rhissa Z., 2010. Revue du secteur de l'élevage au Niger. Ministère de l'Élevage, des Pêches et des Industries Animales. Niger, $115 \mathrm{p}$.

Souvenir Z.P., Zeuh V., Moazami G.K., Laloë D., Bourzat D., Idriss A. et Grosclaude F., 1999. Étude du statut phylogénétique du bovin Kouri du lac Tchad à l'aide de marqueurs moléculaires. Rév. Elev. Méd. Vét. Pays trop. 52 : 155-162.

Souvenir Z.P. et Zeuh V., 1996. Volet des travaux génétiques. In : Projet de « conservation de la race bovine Kouri dans le bassin conventionnel du Lac Tchad», rapport final. N'Djamena. Tchad, LRVZ de Farcha, 16-66.

Tawah C.L., Rege. J.E.O., Aboagye G.S., 1997. A close look at a rare African breed-the Kuri cattle of Lake Chad basin: origin, distribution, production and adaptative characteristics. S. Afr. J. Anim. Sci. 27 (2): 31-40. 
Tchad. Ministère de l'élevage, 2003. Rapport National sur les ressources zoo-génétiques. Ministère de l'élevage.- LRVZ, Farcha, 77p.

Tellah M., ZeuhV., Mopaté L.Y., Mbaïndingatoloum F.M., Boly H., 2015. Paramètres de reproduction des vaches Kouri au Lac Tchad. Journal of Applied Biosciences $90: 8387-8396$.

Zangui I.M.S., 1986. L'élevage des bovins, ovins, caprins au Niger : Étude ethnologique. Thèse vétérinaire de l'EISMV de Dakar,111 p.

Zeuh V., Mopaté L.Y., Youssouf A.I., Djidingar D., 2014. Milk production performance of Kuri Cows under extensive breeding conditions of Lake Chad. International Journal of Agriculture Innovations and Research (IJAIR), 3(3) ISSN (Online): 2319-1473. 